\title{
DISENTANGLEMENT VIA ENTANGLEMENT: A UNIFIED METHOD FOR WANNIER LOCALIZATION
}

\author{
ANIL DAMLE * AND LIN LIN †
}

\begin{abstract}
The Wannier localization problem in quantum physics is mathematically analogous to finding a localized representation of a subspace corresponding to a nonlinear eigenvalue problem. While Wannier localization is well understood for insulating materials with isolated eigenvalues, less is known for metallic systems with entangled eigenvalues. Currently, the most widely used method for treating systems with entangled eigenvalues is to first obtain a reduced subspace (often referred to as disentanglement) and then to solve the Wannier localization problem by treating the reduced subspace as an isolated system. This is a multi-objective nonconvex optimization procedure and its solution can depend sensitively on the initial guess. We propose a new method to solve the Wannier localization problem, avoiding the explicit use of an an optimization procedure. Our method is robust, efficient, relies on few tunable parameters, and provides a unified framework for addressing problems with isolated and entangled eigenvalues.
\end{abstract}

Key words. Wannier functions, Localization, Compression, Density matrix, Band structure, Disentanglement

AMS subject classifications. 65Z05, 82D25, 65F30

1. Introduction. Localized representations of electronic wavefunctions have a wide range of applications in quantum physics, chemistry, and materials science. They require significantly less memory to store, and are the foundation of so-called "linear scaling methods" $[4,16,26]$ for solving quantum problems. They can also be used to analyze the chemical bonding in complex materials, interpolate the band structure of crystals, accelerate ground and excited state electronic structure calculations, and form reduced order models for strongly correlated many body systems [30].

In an effective single particle theory such as the Kohn-Sham density functional theory (KSDFT) [21, 27], the electronic wavefunctions are given by the (possibly generalized) eigenfunctions, denoted $\left\{\psi_{i}(\mathbf{r})\right\}$, of a self-adjoint Hamiltonian operator $\mathcal{H}$ :

$$
\mathcal{H} \psi_{i}(\mathbf{r})=\varepsilon_{i} \psi_{i}(\mathbf{r}), \quad \varepsilon_{i} \in \mathcal{I}
$$

Here $\mathcal{I}$ is an interval that can be interpreted as an energy window that indicates the eigenfunctions of physical interest. These eigenfunctions are generally delocalized, i.e. have significant magnitude in large portions of the computational domain. The Wannier localization problem is as follows: find an approximately minimal set of orthonormal and localized functions $\left\{w_{j}\right\}$, which have significant magnitude on only a small portion of the computational domain, such that

$$
\operatorname{span}\left\{\psi_{j}\right\}_{\varepsilon_{i} \in \mathcal{I}} \subseteq \mathcal{V}_{w}:=\operatorname{span}\left\{w_{j}\right\} .
$$

\footnotetext{
*Department of Computer Science, Cornell University, Ithaca, NY 14853 (damle@cornell.edu).

Funding: National Science Foundation Mathematical Sciences Postdoctoral Research Fellowship (grant number DMS-1606277).

$\dagger$ Department of Mathematics, University of California, Berkeley, Berkeley, CA 94720; and Computational Research Division, Lawrence Berkeley National Laboratory, Berkeley, CA 94720 (linlin@math.berkeley.edu).

Funding: U.S. Department of Energy (contract number DE-SC0017867, the DOE Center for Applied Mathematics for Energy Research Applications (CAMERA) program), National Science Foundation (grant number DMS-1652330) and Alfred P. Sloan fellowship.
} 
Following the convention in quantum physics, $\left\{w_{j}\right\}$ are called Wannier functions [3, $25,42]$.

When the eigenvalues in $\mathcal{I}$ are isolated, i.e.

$$
\inf _{\varepsilon_{i} \in \mathcal{I}, \varepsilon_{i^{\prime}} \notin \mathcal{I}}\left|\varepsilon_{i}-\varepsilon_{i^{\prime}}\right|>0,
$$

the Wannier localization problem has been studied extensively in mathematics and physics $[3,5,7,10-14,19,24,25,31,34-37]$. Loosely speaking, for a class of Hamiltonians $\mathcal{H}$, one can construct exponentially localized Wannier functions such that $\mathcal{V}_{w}=\operatorname{span}\left\{\psi_{i}\right\}_{\varepsilon_{i} \in \mathcal{I}}$. The isolation condition is satisfied when treating valence bands of insulating systems.

When the isolation condition (2) is violated, the eigenvalues in $\mathcal{I}$ become entangled. Entangled eigenvalues appear ubiquitously in metallic systems, but also insulating systems when conduction bands or a selected range of valence bands are considered. The problem now becomes significantly more difficult: both identify a subspace $\mathcal{V}_{w}$ that admits a localized basis, and construct such a basis.

The most widely used method to construct localized functions in this scenario is a disentanglement procedure [41]. It first identifies $\mathcal{V}_{w}$ by minimizing a nonlinear "smoothness functional". Then it computes $\left\{w_{i}\right\}$ by minimizing a nonlinear "spread functional" [31]. In both problems the feasible set is nonconvex. While this two step method has been successfully applied to a number of applications [30], there is little mathematical understanding of the disentanglement procedure. Sensitive dependence on the initial guess along with a number of tunable parameters in the optimization formulation gives rise to a number of practical difficulties in using this method. Often, detailed knowledge of the underlying physical system is required to obtain physically meaningful results.

In this manuscript, we propose a unified method to address the Wannier localization problem for both isolated and entangled cases. Instead of an initial "disentanglement" step, our method explicitly constructs a quasi-density matrix that "entangles" the eigenfunctions of interest with the rest of the eigenfunctions in a controlled manner. This has the effect of simultaneously identifying the subspace $\mathcal{V}_{w}$ and constructing the localized basis. For the isolated case our new method reduces to the prior selected columns of the density matrix (SCDM) method [12], and hence we still refer to our new, unified approach as SCDM.

The core technical contributions of this manuscript are the extension of SCDM to the entangled band case through the use of a quasi-density matrix, and a significantly simplified extension to crystal systems than that of prior work [13]. While our methodology can be used to treat a broad range of physical systems, the SCDM algorithm for the isolated case will fail for topological insulators. In this setting, the band structure is isolated but the corresponding isolated bands do not admit well-localized Wannier functions due to topological obstruction [5, 11,40].

The SCDM method has several significant advantages. First is its simplicity. There are essentially no tunable parameters for the isolated case and only two parameters in the entangled case. Second, SCDM is constructed using standard linear algebra operations, which makes it easy to implement and parallelize. Third, SCDM is a deterministic, one-step procedure and does not require an initial guess. Hence, it will not get stuck at local minima as other nonconvex, nonlinear optimization methods may. Finally, SCDM unifies the treatment of molecules and crystals, while standard methods often require a significantly more complex treatment for crystals. We provide an interface to the widely used Wannier90 software package [33] and demonstrate the 
effectiveness of SCDM via several examples of real materials with isolated and entangled eigenvalues.

2. The SCDM method. We consider the effective one-body Schrödinger operator $\mathcal{H}=-\frac{1}{2} \Delta+V(\mathbf{r})$ in $\mathbb{R}^{3}$, and all eigenfunctions of interest $\psi_{i}(\mathbf{r}) \in L^{2}\left(\mathbb{R}^{3}\right)$. This corresponds to problems involving molecules and nanoclusters, which require a simpler setup than our forthcoming discussion of crystals.

For the isolated case, without loss of generality we assume only the algebraically smallest $N$ eigenvalues $\left\{\varepsilon_{i}\right\}_{i=1}^{N}$ are in the interval $\mathcal{I}$, and the corresponding eigenfunctions $\left\{\psi_{i}\right\}_{i=1}^{N}$ are orthonormal. Using Dirac notation, the density matrix

$$
P=\sum_{i=1}^{N}\left|\psi_{i}\right\rangle\left\langle\psi_{i}\right|
$$

is a rank $N$ matrix that is the spectral projector associated with $\mathcal{H}$ onto the interval $\mathcal{I}$. Notably, its kernel $P\left(\mathbf{r}, \mathbf{r}^{\prime}\right)$ decays rapidly as $\left|\mathbf{r}-\mathbf{r}^{\prime}\right| \rightarrow \infty$ (specifics of the decay rates may be found in, e.g., $[2,26])$. Intuitively, if we can select a set of $N$ points $\mathcal{C}=\left\{\mathbf{r}_{i}\right\}_{i=1}^{N}$ so that the corresponding column vectors of the kernel $\left\{P\left(\mathbf{r}, \mathbf{r}_{i}\right)\right\}_{i=1}^{N}$ are the "most representative" and well conditioned column vectors of $P$, these vectors almost form the desired Wannier functions up to the orthonormality condition.

In order to select the set $\mathcal{C}$, we let $\Psi \in \mathbb{C}^{N_{g} \times N}$ denote the unitary matrix corresponding to a discrete representation of $\left\{\psi_{i}(\mathbf{r})\right\}_{i=1}^{N}$ using their nodal values on $N_{g}$ grid points ${ }^{1}$. The corresponding discretized density matrix, still denoted by $P$, is given by $P=\Psi \Psi^{*}$. Conceptually, the most representative column vectors can be identified via a QR factorization with column-pivoting (QRCP) [17] applied to $P$. However, this is often impractical since $P$ is prohibitively expensive even to construct and store in memory. The SCDM method [12] leverages the fact that a good set $\mathcal{C}$ can be equivalently computed via the QRCP of the matrix $\Psi^{*}$ (see Remark 1 ) as

$$
\Psi^{*} \Pi=Q R \equiv Q\left[\begin{array}{ll}
R_{1} & R_{2}
\end{array}\right] .
$$

Here $\Pi$ is a permutation matrix, $Q$ is a unitary matrix, $R_{1} \in \mathbb{C}^{N \times N}$ is an upper triangular matrix, and $R_{2} \in \mathbb{C}^{N \times\left(N_{g}-N\right)}$. The points $\mathcal{C}=\left\{\mathbf{r}_{i}\right\}_{i=1}^{N}$ can be directly identified from the first $N$ columns of the permutation matrix $\Pi$.

REMARK 1. Under the assumption that the QRCP is computed via the algorithm by Golub and Businger [6], in exact arithmetic the permutation matrices $\Pi$ computed for factorizations of $P$ and $\Psi^{*}$ will be identical. However, there exist other algorithms for computing so-called rank-revealing $Q R$ factorizations [8, 18]. Therefore, the more relevant aspect of $S C D M$ is that if a good rank-revealing $Q R$ factorization $\Psi^{*} \Pi=Q\left[\begin{array}{ll}R_{1} & R_{2}\end{array}\right]$ is computed-in that $R_{1}$ is well conditioned-we have a good rank-revealing $Q R$ factorization of $P$ as $P \Pi=(\Psi Q)\left[\begin{array}{ll}R_{1} & R_{2}\end{array}\right]$. This justifies our use of $\Psi^{*}$ independent of the actual algorithm used for the rank-revealing $Q R$ factorization.

Having chosen $\mathcal{C}$, we must now orthonormalize the localized column vectors $\left\{P\left(\mathbf{r}, \mathbf{r}_{i}\right)\right\}_{i=1}^{N}$ without destroying their locality. Note that

$$
P\left(\mathbf{r}, \mathbf{r}_{i}\right)=\sum_{i^{\prime}=1}^{N} \psi_{i^{\prime}}(\mathbf{r}) \Xi_{i^{\prime}, i}
$$

\footnotetext{
${ }^{1}$ We are implicitly assuming $N_{g} \geq N$, and since we have discretized $\mathbf{r}$ the set $\mathcal{C}$ will correspond to picking $N$ of the $N_{g}$ points to define the columns.
} 
where $\Xi \in \mathbb{C}^{N \times N}$ has matrix elements $\Xi_{i, i^{\prime}}=\psi_{i}^{*}\left(\mathbf{r}_{i^{\prime}}\right)$. One way to enforce the orthogonality is to define

$$
w_{i}(\mathbf{r})=\sum_{i^{\prime}=1}^{N} \psi_{i^{\prime}}(\mathbf{r}) U_{i^{\prime}, i}, \quad U=\Xi\left(\Xi^{*} \Xi\right)^{-\frac{1}{2}} .
$$

Here $U \in \mathbb{C}^{N \times N}$ is a unitary matrix and is referred to as a gauge in the physics literature. The matrix square root transformation in Eq. (4) is called the Löwdin transformation [29] and may be equivalently computed using the orthogonal factors from the reduced SVD of $\Xi$.

Considering

$$
\left(\Xi^{*} \Xi\right)_{i, i^{\prime}}=\sum_{i^{\prime \prime}=1}^{N} \psi_{i^{\prime \prime}}\left(\mathbf{r}_{i}\right) \psi_{i^{\prime \prime}}^{*}\left(\mathbf{r}_{i^{\prime}}\right)=P\left(\mathbf{r}_{i}, \mathbf{r}_{i^{\prime}}\right),
$$

the decay properties of $P$ imply that $\left[P\left(\mathbf{r}_{i}, \mathbf{r}_{i^{\prime}}\right)\right]$ may be viewed as a localized $N \times N$ matrix. If the eigenvalues of $\left(\Xi^{*} \Xi\right)^{-\frac{1}{2}}$ are bounded from below by a positive value, then $\left(\Xi^{*} \Xi\right)^{-\frac{1}{2}}$ will itself be localized [2], and consequently $\left\{w_{i}\right\}_{i=1}^{N}$ will be localized, orthonormal Wannier functions.

REMARK 2. Numerical observations indicate that for many real materials, the eigenvalues of $\left(\Xi^{*} \Xi\right)^{-\frac{1}{2}}$ are indeed bounded from below by a positive value. Furthermore, the condition number of this matrix can be very close to 1 in practice. However, it is known that topological insulators (see, e.g., [9, 20]) have isolated band structure but do not admit exponentially localized Wannier functions. Hence, there must necessarily be a failure mode of our algorithm. In fact, for the topologically nontrivial Kane-Mele model [23] numerical experiments have shown that, when restricted to the occupied bands, irrespective of the column set used $\Xi^{*}(\mathbf{k}) \Xi(\mathbf{k})$ in Eq. (19) will become singular for some $\mathbf{k}$ in the Brillouin zone [40]. Therefore, the SCDM method, predicated on being able to choose columns good for all $\mathbf{k}$, necessarily fails. Such a statement holds generally for topological insulators, such as Chern insulators and $\mathbb{Z}_{2}$ insulators [5,11].

For the entangled case, we extend the SCDM method by "entangling" the eigenfunctions of interest with additional eigenfunctions through the use of a quasi-density matrix

$$
P=\sum_{i}\left|\psi_{i}\right\rangle f\left(\varepsilon_{i}\right)\left\langle\psi_{i}\right|=f(H)
$$

where $f(\cdot)$ is a smooth function, $\mathcal{I}$ is a subset of the support set of $f$, and the summation is formally over all eigenfunctions of $\mathcal{H}$. From this perspective, the case of isolated band is associated with the choice $f(\varepsilon)=\mathbf{1}_{\mathcal{I}}(\varepsilon)$, the indicator function on the interval $\mathcal{I}$.

We now assume there is a number $\mu_{c}$ such that $\inf _{i}\left|\varepsilon_{i}-\mu_{c}\right|$ is very small or even zero. The following two scenarios of entangled eigenvalues appear most frequently in quantum physics, corresponding to the Wannier localization problem below and around a certain energy level (usually the Fermi energy) respectively [43]. In both cases $f(\varepsilon)$ is large on the region of interest and smoothly decays to zero outside $\mathcal{I}$ in a manner controlled by a parameter $\sigma$ (see Fig. 1). 

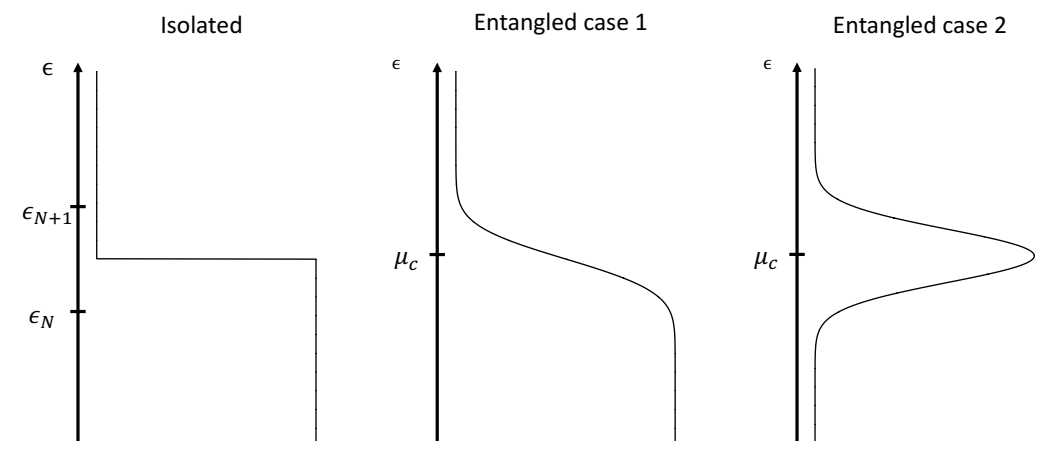

Fig. 1: $f(\varepsilon)$ for the isolated and two entangled cases.

Entangled case 1: $\mathcal{I}=\left(-\infty, \mu_{c}\right)$. In this case we can choose a value $\sigma>0$ and let

$$
f(\varepsilon)=\frac{1}{2} \operatorname{erfc}\left(\frac{\varepsilon-\mu_{c}}{\sigma}\right)=\frac{1}{\sqrt{\pi \sigma^{2}}} \int_{\varepsilon}^{\infty} \exp \left(-\frac{\left(t-\mu_{c}\right)^{2}}{\sigma^{2}}\right) \mathrm{d} t .
$$

The function $f(\varepsilon)$ satisfies $\lim _{\varepsilon \rightarrow-\infty} f(\varepsilon)=1, \lim _{\varepsilon \rightarrow \infty} f(\varepsilon)=0$ and the transition occurs smoothly around $\mu_{c}$.

Entangled case 2: $\mathcal{I}=\left(\mu_{c}-\sigma, \mu_{c}+\sigma\right)$. In this case we choose $f$ to be a Gaussian function

$$
f(\varepsilon)=\exp \left(-\frac{\left(\varepsilon-\mu_{c}\right)^{2}}{\sigma^{2}}\right)
$$

In both cases $f(\varepsilon)$ exhibits the smoothness and decay properties that we need, but the choice of $f(\varepsilon)$ is certainly not unique. For instance, in the entangled case 1 we may use the Fermi-Dirac function instead. Nevertheless, numerical results indicate that the present choices perform well in practice.

For a smooth function $f$, the kernel of the quasi-density matrix $P\left(\mathbf{r}, \mathbf{r}^{\prime}\right)$ also decays rapidly ${ }^{2}$. Given a desired number of Wannier functions $N_{w}{ }^{3}$ we would once again like to select $N_{w}$ "most representative" and well conditioned column vectors of $P$ to construct them. Let $\mathcal{E}=\operatorname{diag}\left[\left\{\varepsilon_{i}\right\}\right] \in \mathbb{R}^{N \times N}$ be a diagonal matrix containing all eigenvalues such that $f(\varepsilon)$ is above some threshold, and $\Psi \in \mathbb{C}^{N_{g} \times N}$ be the matrix containing the corresponding discretized eigenvectors. We can now compute a QRCP for the weighted eigenvectors

$$
\left(f(\mathcal{E}) \Psi^{*}\right) \Pi=Q R
$$

and select the $N_{w}$ columns corresponding to the left most $N_{w}$ columns of the permutation $\Pi$. As before, we let $\mathcal{C}=\left\{\mathbf{r}_{i}\right\}_{i=1}^{N_{w}}$ denote the real space points corresponding to the selected columns and define the auxiliary matrix $\Xi \in \mathbb{C}^{N \times N_{w}}$ with $\Xi_{i, i^{\prime}}=f\left(\varepsilon_{i}\right) \psi_{i}^{*}\left(\mathbf{r}_{i^{\prime}}\right)$.

\footnotetext{
${ }^{2}$ Here we deliberately omit the discussion on the decay rate in order to unify the discussion for molecular and crystal systems. For the molecular case here, we may apply the theoretical statements in e.g. $[2,28]$

${ }^{3}$ For metallic systems this is often set to be equal to the number of bands plus a small integer, a heuristic recently justified mathematically [10]
} 
If the eigenvalues of $\Xi^{*} \Xi$ are bounded away from 0 , the choice of gauge $U=\Xi\left(\Xi^{*} \Xi\right)^{-\frac{1}{2}}$ once again gives rise to the Wannier functions. Now, $U \in \mathbb{C}^{N \times N_{w}}$ is a rectangular matrix with orthonormal columns. Fig. 2 compares the delocalized eigenfunctions and the localized Wannier functions corresponding to isolated and entangled cases using a simple one-dimensional model problem, the details of which may be found in Appendix B.

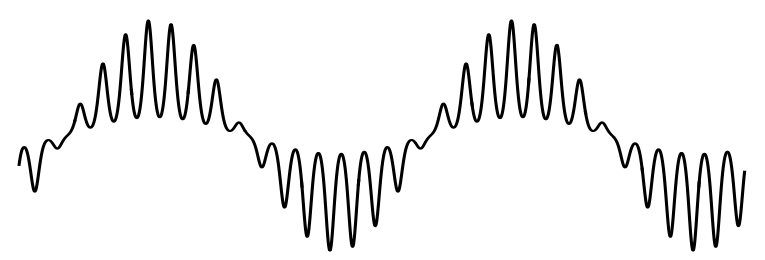

(a)

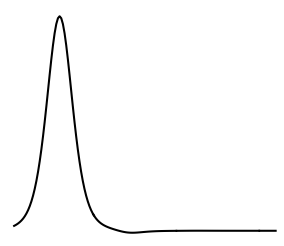

(b)

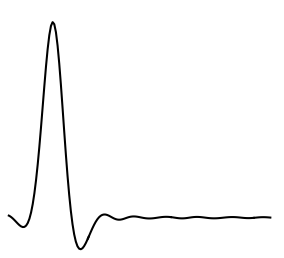

(c)

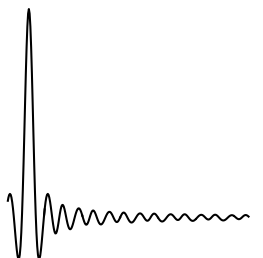

(d)

Fig. 2: Eigenfunctions and computed Wannier functions for a simple one-dimensional model problem. (a) An example eigenfunction plotted on the whole domain, (b) the isolated case, (c) entangled case 1, and (d) entangled case 2. For the three examples of functions computed by the SCDM method we have zoomed in on the region where the bulk of the function is supported.

3. Bloch-Floquet theory. To facilitate further discussion we briefly review Bloch-Floquet theory for crystal structures. Without loss of generality we consider a three-dimensional crystal. The Bravais lattice with lattice vectors $\mathbf{a}_{1}, \mathbf{a}_{2}, \mathbf{a}_{3} \in \mathbb{R}^{3}$ is defined as

$$
\mathbb{L}=\left\{\mathbf{R} \mid \mathbf{R}=n_{1} \mathbf{a}_{1}+n_{2} \mathbf{a}_{2}+n_{3} \mathbf{a}_{3}, \quad n_{1}, n_{2}, n_{3} \in \mathbb{Z}\right\} .
$$

The the potential $V$ is real-valued and $\mathbb{L}$-periodic, i.e.

$$
V\left(\mathbf{r}+n_{i} \mathbf{a}_{i}\right)=V(\mathbf{r}), \quad \forall \mathbf{r} \in \mathbb{R}^{3}, n_{i} \in \mathbb{Z} .
$$

The unit cell is defined as

$$
\Gamma=\left\{\mathbf{r}=c_{1} \mathbf{a}_{1}+c_{2} \mathbf{a}_{2}+c_{3} \mathbf{a}_{3} \mid-1 / 2 \leq c_{1}, c_{2}, c_{3}<1 / 2\right\} .
$$

The Bravis lattice induces a reciprocal lattice $\mathbb{L}^{*}$, and the unit cell of the reciprocal lattice is called the (first) Brillouin zone and denoted by $\Gamma^{*}$. The problem formulation in the previous section can be formally identified as a special case of this setup with an infinitely large unit cell. 
According to the Bloch-Floquet theory, the spectrum of $\mathcal{H}$ can be relabeled using two indices $(b, \mathbf{k})$, where $b \in \mathbb{N}$ is called the band index and $\mathbf{k} \in \Gamma^{*}$ is the Brillouin zone index. Each generalized eigenfunction $\psi_{b, \mathbf{k}}(\mathbf{r})$ is known as a Bloch orbital and satisfies $\mathcal{H} \psi_{b, \mathbf{k}}(\mathbf{r})=\varepsilon_{b, \mathbf{k}} \psi_{b, \mathbf{k}}(\mathbf{r})$ with periodic boundary conditions. Furthermore, $\psi_{b, \mathbf{k}}$ can be decomposed as $\psi_{b, \mathbf{k}}(\mathbf{r})=e^{\imath \mathbf{k} \cdot \mathbf{r}} u_{b, \mathbf{k}}(\mathbf{r})$, where $u_{b, \mathbf{k}}(\mathbf{r})$ is a periodic function with respect to $\mathbb{L}$. An eigenpair $\left(\varepsilon_{b, \mathbf{k}}, u_{b, \mathbf{k}}\right)$ can be obtained by solving the eigenvalue problem

$$
\mathcal{H}(\mathbf{k}) u_{b, \mathbf{k}}=\varepsilon_{b, \mathbf{k}} u_{b, \mathbf{k}}(\mathbf{r}), \quad \mathbf{r} \in \Gamma, \quad \mathbf{k} \in \Gamma^{*},
$$

where $\mathcal{H}(\mathbf{k})=-\frac{1}{2}(\nabla+\imath \mathbf{k})^{2}+V(\mathbf{r})$. For each $\mathbf{k}$, the eigenvalues $\varepsilon_{b, \mathbf{k}}$ are ordered non-decreasingly. For a fixed $b,\left\{\varepsilon_{b, \mathbf{k}}\right\}$ as a function of $\mathbf{k}$ is called a Bloch band. The collection of all eigenvalues are called the band structure of the crystal, which characterizes the spectrum of the operator $\mathcal{H}$. In this framework, the isolation condition (2) becomes

$$
\inf \left|\varepsilon_{b, \mathbf{k}}-\varepsilon_{b^{\prime}, \mathbf{k}^{\prime}}\right|>0, \quad \mathbf{k}, \mathbf{k}^{\prime} \in \Gamma^{*}, \varepsilon_{b, \mathbf{k}} \in \mathcal{I}, \varepsilon_{b^{\prime}, \mathbf{k}^{\prime}} \notin \mathcal{I} \text {. }
$$

4. Wannier functions for crystals and disentanglement. Mirroring our prior discussion, we first we consider the isolated case. Without loss of generality we assume the eigenvalues in $\mathcal{I}$ are labeled as $\left\{\varepsilon_{b, \mathbf{k}}\right\}_{b=1}^{N_{b}}$. If we rotate $\left\{\psi_{b, \mathbf{k}}\right\}$ by an arbitrary unitary matrix, now indexed by $\mathbf{k}, U(\mathbf{k}) \in \mathbb{C}^{N_{b} \times N_{b}}$, we can define a new set of functions

$$
\widetilde{\psi}_{b, \mathbf{k}}(\mathbf{r})=\sum_{b^{\prime}=1}^{N_{b}} \psi_{b^{\prime}, \mathbf{k}}(\mathbf{r}) U_{b^{\prime}, b}(\mathbf{k}), \quad \mathbf{k} \in \Gamma^{*} .
$$

A given set of matrices $\{U(\mathbf{k})\}$ is called a Bloch gauge. For any choice of Block gauge, the Wannier functions for crystals are [42]

$$
w_{b, \mathbf{R}}(\mathbf{r})=\frac{1}{\left|\Gamma^{*}\right|} \int_{\Gamma^{*}} \widetilde{\psi}_{b, \mathbf{k}}(\mathbf{r}) e^{-\imath \mathbf{k} \cdot \mathbf{R}} \mathrm{d} \mathbf{k}, \quad \mathbf{r} \in \mathbb{R}^{3}, \mathbf{R} \in \mathbb{L},
$$

where $\left|\Gamma^{*}\right|$ is the volume of the Brillouin zone. For a class of $\mathcal{H}$ there exists a gauge such that $\widetilde{\psi}_{b, \mathbf{k}}$ is analytic in $\mathbf{k}$, implying that each Wannier function decays exponentially as $|\mathbf{r}| \rightarrow \infty[3,37]$. Furthermore, the set of Wannier functions $\left\{w_{b, \mathbf{R}}(\mathbf{r})\right\}$ forms an orthonormal basis of the subspace in $L^{2}\left(\mathbb{R}^{3}\right)$ spanned by the Bloch orbitals associated with eigenvalues in $\mathcal{I}$. For crystals, the Wannier localization problem is thus partly reduced to the problem of finding a gauge such that $\widetilde{\psi}_{b, \mathbf{k}}$ is smooth with respect to k. This can be done by minimizing the "spread functional" [31]

$$
\Omega\left[\left\{w_{b, \mathbf{0}}\right\}_{b=1}^{N_{b}}\right]=\sum_{b=1}^{N} \int\left|w_{b, \mathbf{0}}(\mathbf{r})^{2}\right| \mathbf{r}^{2} \mathrm{~d} \mathbf{r}-\left(\int\left|w_{b, \mathbf{0}}(\mathbf{r})^{2}\right| \mathbf{r} \mathrm{d} \mathbf{r}\right)^{2} .
$$

Only $\mathbf{R}=\mathbf{0}$ is considered because Wannier functions associated with different $\mathbf{R}$ 's only differ by translation.

In the entangled case, the disentanglement method constructs the gauge via a twostep procedure. It first finds a gauge $U^{\text {dis }}(\mathbf{k}) \in \mathbb{C}^{N_{b} \times N_{w}}$, in order to disentangle the given set of $N_{b}$ functions into $N_{w}$ functions for each $\mathbf{k}$. This is obtained by minimizing a "smoothness functional" (the gauge invariant part of the spread functional of $\Omega$, see $[30,41]$ for details) with respect to $\mathbf{k}$ for the following auxiliary functions

$$
\psi_{b, \mathbf{k}}^{\mathrm{dis}}(\mathbf{r})=\sum_{b^{\prime}=1}^{N_{b}} \psi_{b^{\prime}, \mathbf{k}}(\mathbf{r}) U_{b^{\prime}, b}^{\mathrm{dis}}(\mathbf{k}), \quad b=1, \ldots, N_{w}, \mathbf{k} \in \Gamma^{*} .
$$


After obtaining $U^{\text {dis }}(\mathbf{k})$ a second gauge $U^{\text {loc }}(\mathbf{k}) \in \mathbb{C}^{N_{w} \times N_{w}}$ for each $\mathbf{k}$ is computed by minimizing the spread functional (16). Finally, the overall gauge in the disentanglement method is the composition $U(\mathbf{k})=U^{\text {dis }}(\mathbf{k}) U^{\text {loc }}(\mathbf{k})$.

This two-step procedure can be viewed as a heuristic means to solve a nonlinear, nonconvex multi-objective optimization problem aiming to simultaneously maximize the smoothness functional with respect to $\mathbf{k}$, and minimize the spread functional. Our numerical results indicate that, at least in some cases, this two step procedure may not be an effective surrogate for the desired optimization problem.

5. SCDM for crystals. We now proceed to discuss the relatively minor modifications needed to generalize the SCDM method to crystals.

In the isolated case, for each $\mathbf{k}$-point in the Brillouin zone the $\mathbf{k}$-dependent density matrix is gauge invariant

$$
P(\mathbf{k})=\sum_{\varepsilon_{b, \mathbf{k}} \in \mathcal{I}}\left|\psi_{b, \mathbf{k}}\right\rangle\left\langle\psi_{b, \mathbf{k}}\left|=\sum_{\varepsilon_{b, \mathbf{k}} \in \mathcal{I}}\right| \widetilde{\psi}_{b, \mathbf{k}}\right\rangle\left\langle\widetilde{\psi}_{b, \mathbf{k}}\right|,
$$

and is already an analytic function of $\mathbf{k}[35,37]$. The SCDM method uses the density matrix to construct a gauge so that $\widetilde{\psi}_{b, \mathbf{k}}$ is smooth with respect to $\mathbf{k}$. However, for crystals, we need to select a common set of columns for all the k-dependent density matrices. Previous work [13] suggests that it is often sufficient to select the columns using an "anchor" point $\mathbf{k}_{0}$, such as the so-called Gamma-point $(0,0,0)^{T}$ to identify these columns. A generalization of this procedure is also outlined in [13], though in our numerical experiments we have found use of the Gamma-point as the anchor point suffices.

Let $\Psi_{\mathbf{k}} \in \mathbb{C}^{N_{g} \times N_{b}}$ be the unitary matrix representing $\left\{\psi_{b, \mathbf{k}}(\mathbf{r})\right\}$ on a discrete grid in the unit cell. At the anchor point $\mathbf{k}_{0}$, we compute the QRCP

$$
\Psi_{\mathbf{k}_{0}}^{*} \Pi=Q R \text {. }
$$

As before, let $\mathcal{C}=\left\{\mathbf{r}_{b}\right\}_{b=1}^{N_{b}}$ denote the grid points corresponding to the $N_{b}$ selected columns where $\mathbf{r}_{b} \in \Gamma$. For each $\mathbf{k}$ point, define the auxiliary matrix $\Xi \in \mathbb{C}^{N_{b} \times N_{b}}$ with matrix elements $\Xi_{b, b^{\prime}}(\mathbf{k})=\psi_{b, \mathbf{k}}^{*}\left(\mathbf{r}_{b^{\prime}}\right)$. Then the smoothness of the density matrix $P(\mathbf{k})$ implies that each function

$$
P_{b, \mathbf{k}}(\mathbf{r})=\sum_{b^{\prime}=1}^{N_{b}} \psi_{b^{\prime}, \mathbf{k}}(\mathbf{r}) \Xi_{b^{\prime}, b}(\mathbf{k})
$$

is smooth with respect to $\mathbf{k}$. As before the SCDM gauge can be constructed via the Löwdin transformation as

$$
U(\mathbf{k})=\Xi(\mathbf{k})\left[\Xi^{*}(\mathbf{k}) \Xi(\mathbf{k})\right]^{-\frac{1}{2}} .
$$

Similar to Eq. (5),

$$
\left(\Xi^{*}(\mathbf{k}) \Xi(\mathbf{k})\right)_{b, b^{\prime}}=\sum_{b^{\prime \prime}=1}^{N_{b}} \psi_{b^{\prime \prime}, \mathbf{k}}\left(\mathbf{r}_{b}\right) \psi_{b^{\prime \prime}, \mathbf{k}}^{*}\left(\mathbf{r}_{b^{\prime}}\right)=P\left(\mathbf{r}_{b}, \mathbf{r}_{b^{\prime}} ; \mathbf{k}\right) .
$$

Since the kernel $P\left(\mathbf{r}_{b}, \mathbf{r}_{b^{\prime}} ; \mathbf{k}\right)$ is smooth with respect to $\mathbf{k}$, a sufficient condition for the matrix $\left(\Xi^{*}(\mathbf{k}) \Xi(\mathbf{k})\right)^{-\frac{1}{2}}$ to be smooth with respect to $\mathbf{k}$ is that $\left\|\Xi^{*}(\mathbf{k}) \Xi(\mathbf{k})-I\right\|_{2} \leq \frac{1}{2}$ for all $\mathbf{k}$ in the Brillouin zone. As discussed before, numerical observations for many real materials indicate that this condition holds, but would fail in the context of topological insulators. 
REMARK 3. Although the SCDM algorithm for the isolated case would fail for topological insulators, when the Hamiltonian satisfies the time-reversal symmetry, it is still of interest to modify the SCDM method to select "generalized columns" to construct well localized Wannier functions that do not obey the time-reversal symmetry [11]. Another interesting possibility for treating general topological insulators is to increase the number of Wannier functions and use the algorithm for the entangled case. This will be investigated in the future.

As a result,

$$
\widetilde{\psi}_{b, \mathbf{k}}(\mathbf{r})=\sum_{b^{\prime}=1}^{N_{b}} \psi_{b^{\prime}, \mathbf{k}}(\mathbf{r}) U_{b^{\prime}, b}(\mathbf{k})=\sum_{b^{\prime}=1}^{N_{b}} P_{b^{\prime}, \mathbf{k}}(\mathbf{r})\left[\Xi^{*}(\mathbf{k}) \Xi(\mathbf{k})\right]_{b^{\prime}, b}^{-\frac{1}{2}}
$$

is smooth with respect to $\mathbf{k}$. Therefore, the Fourier transform of $\widetilde{\psi}_{b, \mathbf{k}}$ with respect to $\mathbf{k}$ as in Eq. (15) gives rise to the Wannier functions for crystals constructed by the SCDM method.

In the entangled case (when (13) is not satisfied), the SCDM method makes use of a $\mathbf{k}$-dependent quasi-density matrix for each $\mathbf{k}$ point:

$$
P(\mathbf{k})=\sum_{\varepsilon_{b, \mathbf{k}}}\left|\psi_{b, \mathbf{k}}\right\rangle f\left(\varepsilon_{b, \mathbf{k}}\right)\left\langle\psi_{b, \mathbf{k}}\right|
$$

Here, the choice of $f(\varepsilon)$ matches what we previously discussed, and depends on the desired $\mathcal{I}$. In particular, the reduction to the isolated case is again the choice $f(\varepsilon)=\mathbf{1}_{\mathcal{I}}(\varepsilon)$, which reduces the $\mathbf{k}$-dependent quasi-density matrix to a $\mathbf{k}$-dependent density matrix. Numerical results indicate that these quasi-density matrices for the two entangled cases are smooth with respect to $\mathbf{k}$.

Let $\mathcal{E}(\mathbf{k})=\operatorname{diag}\left[\left\{\varepsilon_{b, \mathbf{k}}\right\}_{b=1}^{N_{b}}\right]$ be a diagonal matrix for each $\mathbf{k}$ containing eigenvalues such that $f(\varepsilon)$ is larger than some threshold. Computing a QRCP at the anchor point in the Brillouin zone $\mathbf{k}_{0}$, we obtain

$$
f\left(\mathcal{E}\left(\mathbf{k}_{0}\right)\right) \Psi_{\mathbf{k}_{0}}^{*} \Pi=Q R .
$$

Analogously to before, the set of real space points $\mathcal{C}=\left\{\mathbf{r}_{b}\right\}_{b=1}^{N_{w}}$ are given by the left most $N_{w}$ columns of the permutation matrix $\Pi$. Defining the auxiliary matrix $\Xi(\mathbf{k}) \in \mathbb{C}^{N_{b} \times N_{w}}$ with matrix elements

$$
\Xi_{b, b^{\prime}}(\mathbf{k})=f\left(\varepsilon_{b, \mathbf{k}}\right) \psi_{b, \mathbf{k}}^{*}\left(\mathbf{r}_{b^{\prime}}\right)
$$

implies that

$$
P_{b, \mathbf{k}}(\mathbf{r})=\sum_{b^{\prime}} \psi_{b^{\prime}, \mathbf{k}}(\mathbf{r}) \Xi_{b^{\prime}, b}(\mathbf{k})
$$

is smooth with respect to $\mathbf{k}$. If the eigenvalues of $\left[\Xi^{*}(\mathbf{k}) \Xi(\mathbf{k})\right]$ are uniformly bounded away from 0 in the Brillouin zone, the gauge $U(\mathbf{k}) \in \mathbb{C}^{N_{b} \times N_{w}}$ given by Eq. (19) is unitary and via (21) defines $\left\{\widetilde{\psi}_{b, \mathbf{k}}\right\}$ that are smooth with respect to k. Eq. (15) once again yields the desired Wannier functions.

6. Wannier interpolation for band structure. In practical electronic structure calculations, the Brillouin zone needs to be discretized using a finite number of points denoted by the set $\mathcal{K}$. The most widely used discretization scheme is the 
Monkhorst-Pack grid [32], which corresponds to a uniform discretization of $\Gamma^{*}$. However, the band structure $\varepsilon_{b, \mathbf{k}}$ as a function of $\mathbf{k}$ often needs to be computed on finely discretized paths (not necessarily grid aligned) through the Brillouin zone. Because the eigenvalues $\varepsilon_{b, \mathbf{k}}$ are in general only Lipschitz continuous with respect to $\mathbf{k}$ [39], interpolating $\varepsilon_{b, \mathbf{k}}$ for $\mathbf{k} \notin \mathcal{K}$ directly from the eigenvalues computed on the grid $\mathcal{K}$ can result in large interpolation errors. The Wannier interpolation method (see e.g., [30]), makes use of the locality of Wannier functions and can yield both higher quality interpolation for a fixed $\mathcal{K}$ and improved convergence with respect to the number of discretization points in $\mathcal{K}$. We simply pair our localized functions computed via the SCDM method with standard Wannier interpolation techniques to compute band structure.

Observe that the computed gauge (in this case from the SCDM method) rotates the periodic part of the Bloch orbitals as well according to

$$
\widetilde{u}_{b, \mathbf{k}}(\mathbf{r})=\sum_{b^{\prime}} u_{b^{\prime}, \mathbf{k}}(\mathbf{r}) U_{b^{\prime}, b}(\mathbf{k}),
$$

and let $\widetilde{H}(\mathbf{k})$ denote the matrix representation of $\mathcal{H}(\mathbf{k})$ in the basis $\left\{\widetilde{u}_{b, \mathbf{k}}\right\}$. We may then construct the reduced matrix

$$
[\widetilde{H}(\mathbf{k})]_{b, b^{\prime}}=\left\langle\widetilde{u}_{b, \mathbf{k}}|\mathcal{H}(\mathbf{k})| \widetilde{u}_{b^{\prime}, \mathbf{k}}\right\rangle=\left[U^{*}(\mathbf{k}) \mathcal{E}(\mathbf{k}) U(\mathbf{k})\right]_{b, b^{\prime}} .
$$

In particular, if the gauge were the identity matrix then $\widetilde{H}(\mathbf{k})$ would be a diagonal matrix with the eigenvalues at $\mathbf{k}$ on the diagonal. However, as noted earlier interpolation via this representation may be very inaccurate. Rather, we would like to interpolate using matrices whose entries are smoother with respect to $\mathbf{k}$ than the eigenvalues themselves are.

This leads to the use of the Wannier functions in (24). The smoothness of $\widetilde{u}_{b, \mathbf{k}}$ with respect to $\mathbf{k}$ implies that each entries of the matrix $\widetilde{H}$ is also smooth with respect to $\mathbf{k}$. Now, Wannier interpolation is precisely given by Fourier interpolation of $\widetilde{H}(\mathbf{k})$ onto the desired points in $\Gamma^{*}$. More specifically, the Fourier transform of $\widetilde{H}(\mathbf{k})$

$$
\mathfrak{H}(\mathbf{R})=\frac{1}{\left|\Gamma^{*}\right|} \int e^{-\imath \mathbf{k} \cdot \mathbf{R}} \widetilde{H}(\mathbf{k}) \mathrm{d} \mathbf{k}
$$

decays rapidly as $|\mathbf{R}| \rightarrow \infty$. When the Monkhorst-Pack grid is used, $\mathfrak{H}(\mathbf{R})$ can be efficiently approximated as

$$
\mathfrak{H}(\mathbf{R}) \approx \frac{1}{N_{\Gamma^{*}}} \sum_{\mathbf{k} \in \mathcal{K}} e^{-\imath \mathbf{k} \cdot \mathbf{R}} \widetilde{H}(\mathbf{k}) .
$$

Here $N_{\Gamma^{*}}=N_{1} N_{2} N_{3}$ is the total number of k-points. From $\mathfrak{H}(\mathbf{R}), \widetilde{H}(\mathbf{k})$ for any $\mathbf{k} \in \Gamma^{*}$ can be reconstructed as

$$
\widetilde{H}(\mathbf{k})=\sum_{\mathbf{R} \in \mathbb{L}} e^{\imath \mathbf{k} \cdot \mathbf{R}} \mathfrak{H}(\mathbf{R}) .
$$

This matrix is small with size $N_{w} \times N_{w}$, and computing its eigenvalues yields the interpolated band structure $\varepsilon_{b, \mathbf{k}}$ for $\mathbf{k} \in \Gamma^{*}$. The summation over the Bravis lattice in Eq. (27) needs to be truncated. The most natural truncation of the Bravis lattice is the parallelepiped dual to the Monkhorst-Pack grid. However, it has been numerically observed that the truncation with a Wigner-Seitz cell leads to smaller numerical error especially when $\mathcal{N}_{\Gamma^{*}}$ is relatively small [43]. 
7. Interface with Wannier90. The solution of the Wannier localization problem is entirely encapsulated in the Bloch gauge $\{U(\mathbf{k})\}$. This allows us to easily integrate our SCDM method with the widely used Wannier90 [33] software package (available online at www.wannier.org) in a non-intrusive way. Wannier90 requires an initial guess for the gauge, and we may simply provide ours as input using the proper file format. Similarly, we may use existing interfaces between electronic structure software packages and Wannier90 to get the requisite input for our code. To facilitate our forthcoming numerical experiments, we built an interface for our method to Wannier90 and the code is available online $e^{4}$.

This procedure allows us to leverage all the functionalities of Wannier90 directly. For example, we can then either set the number of iterations to zero, which allows us to both compute the spread of our SCDM based Wannier functions and perform Wannier interpolation using our computed gauge, or use our gauge as an initial guess and see if the optimization procedure is able to improve it. In addition, our strategy makes comparisons with Wannier functions computed by the optimization methodology in Wannier90 simple.

8. Numerical results. We now demonstrate the effectiveness of the SCDM method qualitatively and quantitatively using real materials. The electronic structure calculations are performed using the QUANTUM ESPRESSO [15] software package with the PBE exchange-correlation functionals [38].

Qualitatively, we examine the shape of the Wannier functions obtained from SCDM, and compare against minimizer of the spread functional (16) in Wannier90. Quantitatively, we measure the value of the spread functional for Wannier functions obtained from SCDM, as well as the accuracy of band structure interpolation from the Wannier functions for isolated and entangled cases. For these examples, we consider the choice of $N_{b}, N_{w}$, and $N_{g}$ as fixed aspects of the problem instance and therefore the SCDM methodology relies on at most two parameters $-\mu$ and $\sigma^{5}$.

Our first example is a $\mathrm{Cr}_{2} \mathrm{O}_{3}$ crystal with collinear spin polarization. Each unit cell has 92 occupied bands and we are interested in the top 6 valence bands, corresponding to 3 spin-up and 3 spin-down $d$ orbitals for the $\mathrm{Cr}$ atoms. This is a challenging system for Wannier90 due to the existence of multiple local minima in the spread functional and the convergence of existing methods can depend sensitively on the choice of the initial guess. For example, in Fig. 3a, when the initial guess is given by projections corresponding to $\mathrm{d}_{x y}, \mathrm{~d}_{y z}$ and $\mathrm{d}_{x z}$ orbitals respectively, the spread functional decreases from $70.56 \AA^{2}$ to $16.99 \AA^{2}$ within 30 steps. In contrast, when the initial guess is given by $\mathrm{sp}^{2}$ hybridized orbitals the spread functional starts at $193.94 \AA^{2}$ and stops decreasing around $47.13 \AA^{2}$, indicating that the optimization procedure is trapped at a stationary point. On the other hand, starting from the SCDM initial guess, the spread starts at $17.22 \AA^{2}$ and quickly converges to $16.98 \AA^{2}$. Fig. $3 \mathrm{~b}$ plots the atomic configuration and isosurface of a localized spin-up orbital obtained from the SCDM gauge without further Wannier optimization. The SCDM localized orbitals clearly demonstrate $d$ orbital character without relying on a user specified initial guess.

Next, we consider two examples with entangled eigenvalues. Fig. 4a shows band structure interpolation for a Si crystal with 8 localized functions computed from

\footnotetext{
${ }^{4}$ https://github.com/asdamle/SCDM

${ }^{5}$ While formally once could consider the anchor point $\mathbf{k}_{0}$ as a tunable parameter, we have observed that the default choice of using the Gamma-point as the anchor point performs robustly in practice. Similarly, the choice of $f(\varepsilon)$ is dictated by the type of problem that one wishes to solve.
} 


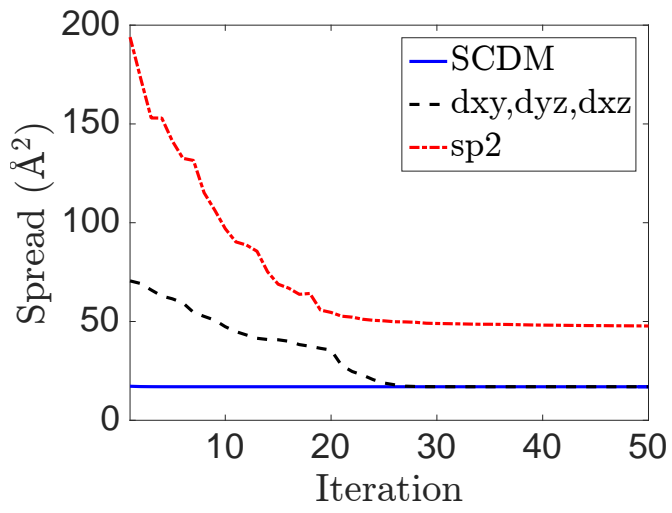

(a)

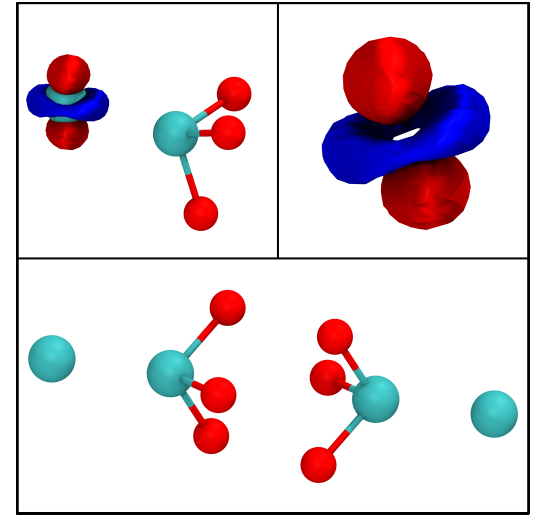

(b)

Fig. 3: (color online) (a) Convergence of the spread for $\mathrm{Cr}_{2} \mathrm{O}_{3}$ starting from the initial guess of the gauge matrix from SCDM (blue solid line), initial guess from d orbitals (black dashed line) and initial guess from $\mathrm{sp}^{2}$ orbitals (red dotted line). (b) One unit cell of $\mathrm{Cr}_{2} \mathrm{O}_{3}$, and the shape of a localized function obtained from SCDM (plot generated using Visual Molecular Dynamics [22]). The localized function has been plotted with and without the molecular structure to better illustrate its character and physical location.

SCDM. This corresponds to entangled case 1, covering both valence bands and lowlying conduction bands. We set $\mu=10.0 \mathrm{eV}, \sigma=2.0 \mathrm{eV}$, and use a $10 \times 10 \times 10 \mathrm{k}$-point grid for constructing the Wannier functions. Fig. $4 \mathrm{~b}$ shows the accuracy of band structure interpolation for a $\mathrm{Cu}$ crystal with 7 localized functions. This corresponds to the entangled case 2, covering valence bands near the Fermi energy contributed mainly from the $d$-orbitals. We use $\mu=15.5 \mathrm{eV}, \sigma=4.0 \mathrm{eV}$, and a $10 \times 10 \times 10 \mathrm{k}$-point grid. In both cases, the SCDM method accurately reproduces the band structure within the energy window of interest.

We now turn to graphene, a metallic system that is particularly challenging for band structure interpolation due to the linear band structure near the Dirac point. Fig. 5 demonstrates that SCDM can accurately interpolate the band structure of graphene even when zooming in on the region near the Dirac point. We set $\mu=-2.5$ $\mathrm{eV}, \sigma=4.0 \mathrm{eV}$, and use a $12 \times 12 \times 1 \mathrm{k}$-grid for constructing the Wannier functions.

Finally, we measure the convergence rate of the band structure interpolation with respect to an increasing number of $\mathbf{k}$-points using Wannier functions obtained from SCDM and those from a (local) minimum corresponding to the optimization objective in Wannier90. Fig. 6a reports the absolute value of the error of the eigenvalues for the occupied bands of $\mathrm{Si}$. The chosen path through the Brillouin zone is discretized with 408 points. A cubic $k \times k \times k$ grid is used and $k$ ranges from 4 to 14 . Both the average and the maximum value of the error converge exponentially with respect to $k$. For the isolated case, the error with the optimized gauge matrix is slightly smaller than that with the SCDM gauge matrix. However, we find that visually the Wannier functions from SCDM with a $6 \times 6 \times 6 \mathrm{k}$-grid already result in excellent band structure.

Fig. $6 \mathrm{~b}$ reports the absolute value of the error of the eigenvalues below the Fermi energy for $\mathrm{Al}$, which is a metallic system with entangled band structure. The chosen 


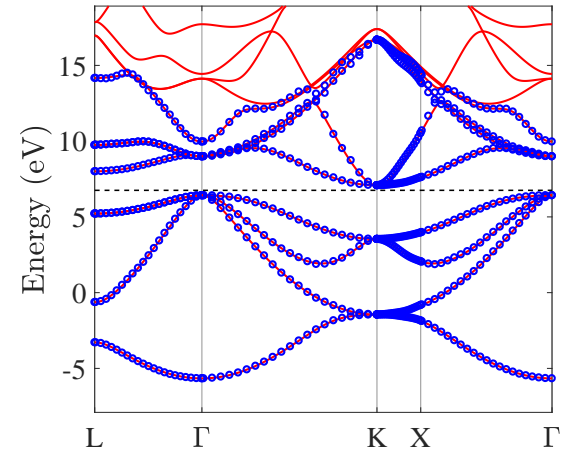

(a)

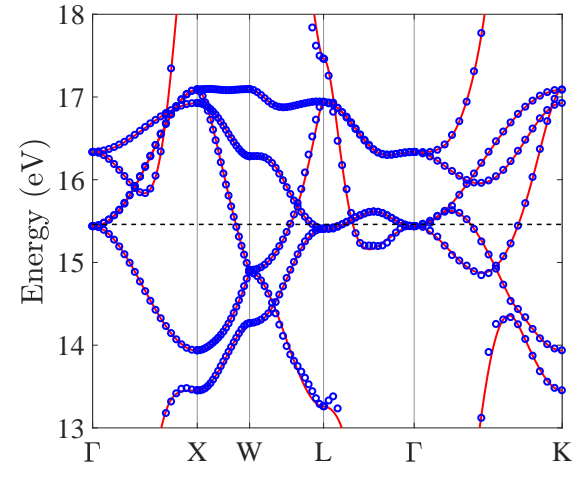

(b)

Fig. 4: (color online) Wannier interpolation with SCDM for the band structure for (a) valance and conduction bands for $\mathrm{Si}(\mathrm{b})$ bands near the Fermi energy for $\mathrm{Cu}$. Direct calculation (red line) and SCDM based Wannier interpolation (blue circles).

path in the Brillouin zone is discretized into 510 points. A cubic $k \times k \times k$ grid is used and $k$ ranges from 6 to 16 . We use the erfc smearing with $\mu$ being the chemical potential at $8.4 \mathrm{eV}$, and $\sigma=4.0 \mathrm{eV}$. We compute six bands for each $\mathbf{k}$ point and SCDM picks the leading four bands. Even for metallic system, numerical results show exponential convergence of the band structure interpolation. Fig. 7a shows that Wannier interpolation using the SCDM gauge matrix with a $10 \times 10 \times 10 \mathrm{k}$ grid already yields excellent band structure. In particular, using the SCDM gauge correctly reproduces band crossings even though a relatively coarse $\mathbf{k}$ grid is used.

For this metallic system, the error of the eigenvalues interpolated using the SCDM gauge matrix is systematically smaller than that of the optimized gauge matrix from Wannier90. Therefore optimization of the spread functional alone does not necessarily improve the interpolation quality. This assessment is further justified by performing Wannier interpolation with a gauge matrix obtained by minimizing the Wannier spread functional directly using six bands and using four orbitals that have $\mathrm{sp}^{3}$ character for the initial guess. In this case the optimized spread is $12.42 \AA^{2}$, while the SCDM gauge gives a larger spread of $18.38 \AA^{2}$. However, Fig. $7 \mathrm{~b}$ shows that the band structure obtained using the optimized gauge with the $\mathrm{sp}^{3}$ initial guess is significantly less accurate when compared to that in Fig. 7a even though the same $\mathbf{k}$ grid is used. In particular, the spread functional alone is not necessarily a proxy for interpolation quality. On the other hand, the SCDM method obtains a smooth density matrix for the range of the required band energies by construction. 


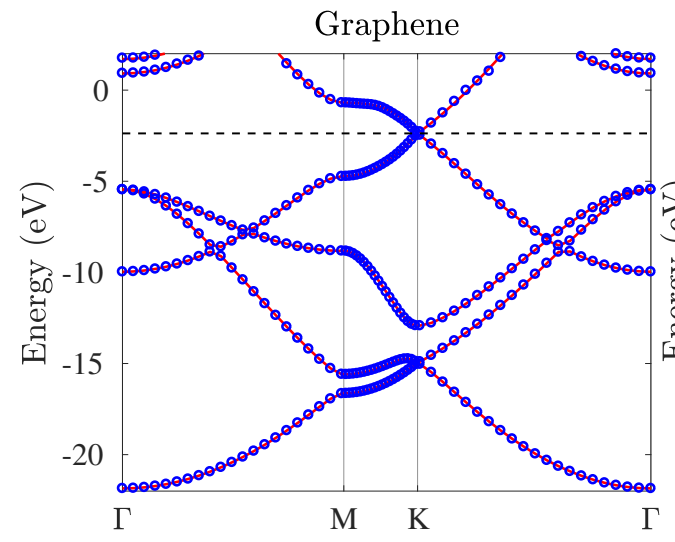

(a)

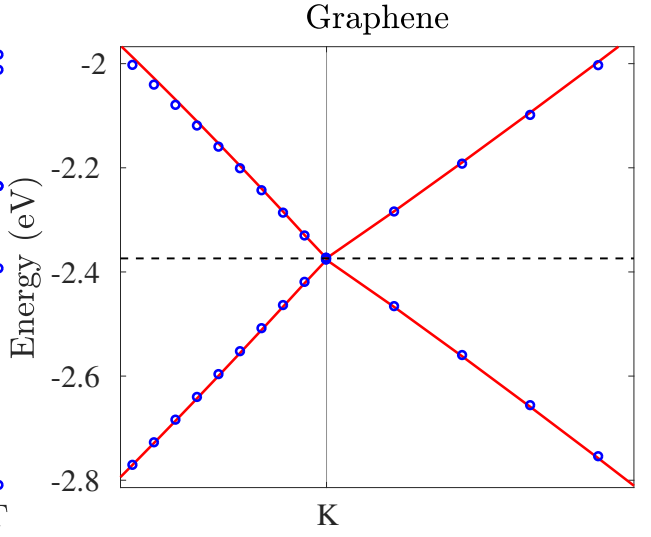

(b)

Fig. 5: (color online) Wannier interpolation with SCDM for the band structure for graphene (a) below the Fermi energy (b) near the Dirac point. Direct calculation (red lines) and SCDM based Wannier interpolation (blue circles).

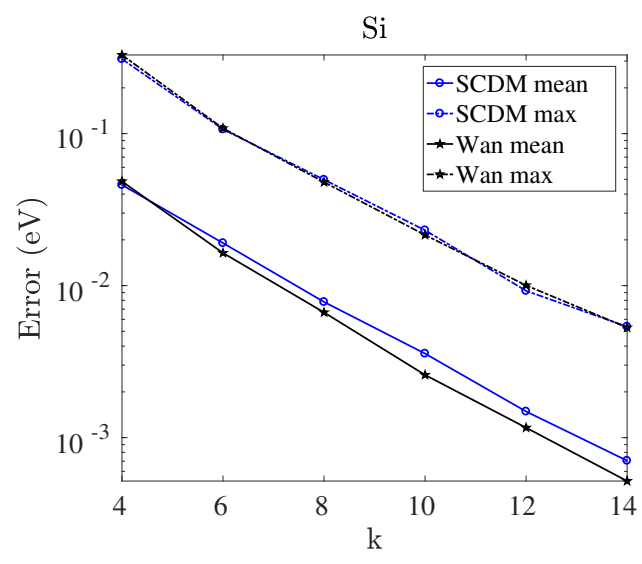

(a)

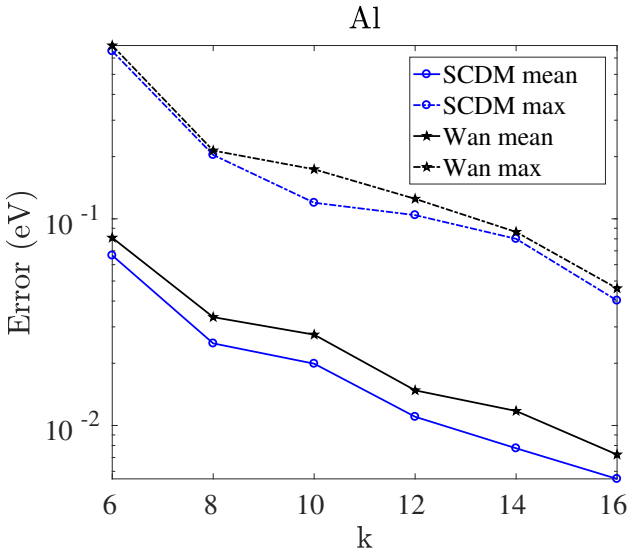

(b)

Fig. 6: (color online) Convergence of the average and maximum error of Wannier interpolation below the Fermi energy using the SCDM gauge matrix, and converged Wannier gauge matrix starting from the SCDM initial guess for (a) silicon (b) aluminum.

9. Discussion and conclusion. We developed a unified method to compute Wannier functions for systems with both isolated and entangled bands. Its simplicity - in both implementation and reliance on few parameters ${ }^{6}$ - makes it easy to use. Of particular importance, our method removes the potentially sensitive dependence

\footnotetext{
${ }^{6}$ Namely, essentially no parameters in the isolated case, and two parameters $\sigma$ and $\mu$ in the entangled case.
} 


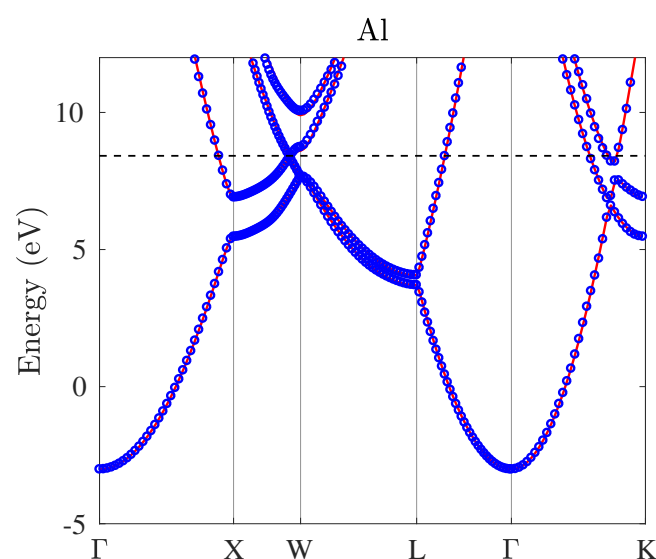

(a)

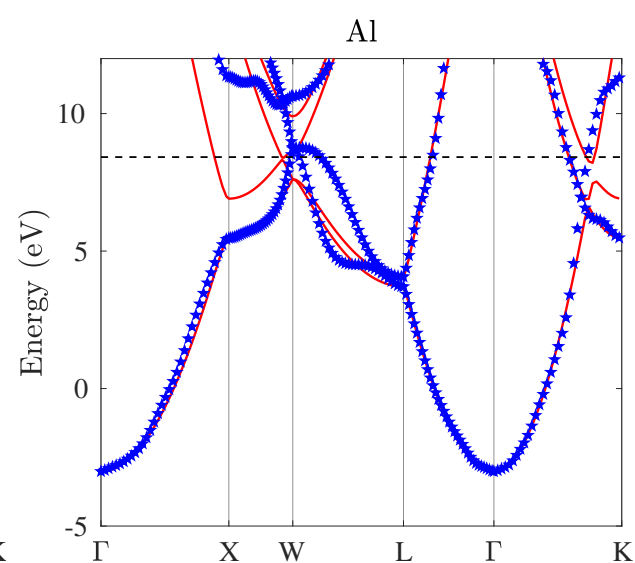

(b)

Fig. 7: (color online) (a) Band structure for $\mathrm{Al}$ around and below the Fermi energy (black dashed line). Direct calculation from QE (red line), and Wannier interpolation using SCDM gauge matrix with a $10 \times 10 \times 10 \mathrm{k}$-grid (blue circle). (b) Same calculation but using the Wannier gauge matrix starting from an $\mathrm{sp}^{3}$ initial guess with $N_{b}=$ $6, N_{w}=4$.

of the construction of Wannier functions on an initial guess to a nonconvex optimization (two-stage in the entangled case) procedure. This potentially makes it easier to treat complicated materials where the choice of a good guess may be difficult, and convergence to local minima may hamper the construction of localized Wannier functions. Interestingly, even though we do not seed our method with a physically informed initial guess, we are able to recover physically interpretable Wannier functions. Furthermore, as we have shown, the objective function of the existing optimization procedure is not necessarily a proxy for good band interpolation. Collectively, these qualities and observations make our new SCDM methodology attractive for the construction of Wannier functions.

Appendix A. Rank-revealing QR factorizations. Our algorithm relies heavily on a QRCP factorization, and therefore we briefly outline standard methodologies for computing these factorizations and briefly discuss the more general class of rankrevealing QR factorizations. Notably, we restrict our discussion to factorizations of matrices that are short, wide, and full row-rank as this is the setting most relevant to this manuscript.

As outlined in Section 2 given an $m \times n$ matrix with $m<n$ and full row-rank we seek to compute a permutation matrix $\Pi$, an $m \times m$ orthogonal matrix $Q$, an $m \times m$ upper triangular matrix $R_{1}$, and a $m \times(n-m)$ matrix $R_{2}$ such that

$$
V \Pi=Q\left[\begin{array}{ll}
R_{1} & R_{2}
\end{array}\right]
$$

where the singular values of $R_{1}$ track those of $V$ as closely as possible. More specifically, we would like a factorization such that

$$
\sigma_{i}\left(R_{1}\right) \geq \frac{\sigma_{i}(V)}{g(n, k)}
$$


for some function $g$ of $n$ and $k$. The specific form of $g(n, k)$ depends on the algorithm used, and there has been significant work on developing algorithms to achieve $g(n, k)$ growing as slowly as possible in $n$ and $k$. We direct the reader to references [8] and [18] for further details.

While more recent rank-revealing algorithms may be necessary for certain problems, often viewed as pathological worst case examples, the most widely used rankrevealing QR factorization is a QR factorization with column pivoting due to Golub and Businger [6]. While formally this algorithm exhibits a rather weak form of $g(n, k)$, its implementation in LAPACK [1] and strong practical performance has driven its use. This practical performance holds true remarkably robustly in our setting and for all the problems we have considered.

The underlying algorithm ${ }^{7}$ is encapsulated by a simple heuristic strategy for picking $\Pi$. This is outlined in Algorithm 1, and can be colloquially summarized as greedily picking columns at each step that look the least like those already selected. At the conclusion of Algorithm 1 the first $m$ entries of $\pi$ yield the information we require about which $m$ columns were selected as pivots during the course of the algorithm, and we have omitted any reference to $Q$ or $\left[\begin{array}{ll}R_{1} & R_{2}\end{array}\right]$ for simplicity. Importantly, we emphasize that Algorithm 1 is a conceptual presentation of the algorithm. In practice an implementation would differ significantly from this description.

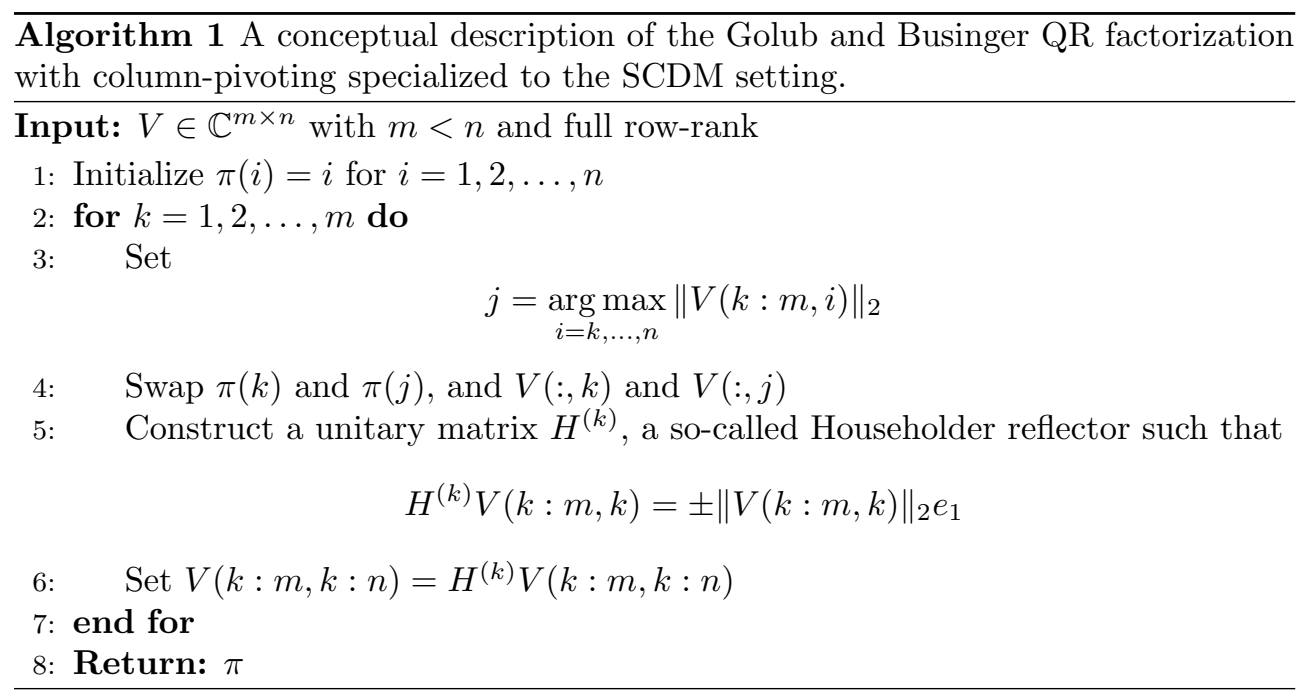

Appendix B. One dimensional model problem. The one dimensional model problem consists of a Hamiltonian operator based on the one-dimensional periodic potential plotted in Figure 8 and discretized on 1280 points. Figure 9 illustrates additional localized functions for each of the three cases. Here we observe that their behavior matches that of the examples shown in the main text. Here, the size of the problem allowed us to computed all 1280 eigenfunctions and only consider the ones of interest. In the first isolated case we set $\mu_{c}=0.2$ and $\sigma=0.5$, and in the second case we set $\mu_{c}=0.15$ and $\sigma=2$. These plots may be reproduced using the included data and files in the code repository available at https://github.com/asdamle/SCDM.

Acknowledgments. The authors thank Stefano Baroni, Eric Cancès, Roberto

\footnotetext{
${ }^{7} \mathrm{~A}$ textbook presentation may be found in Golub and Van Loan [17]
} 


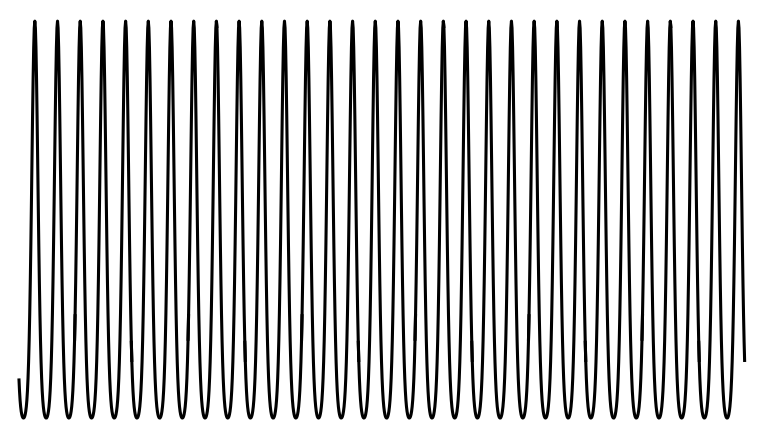

Fig. 8: The potential function for our one dimensional model problem.

Car, Sinisa Coh, Wibe de Jong, Antoine Levitt, Jianfeng Lu, Nicola Marzari, Lukas Müchler and Lexing Ying for useful discussions; Sinisa Coh for providing the atomic structure for the $\mathrm{Cr}_{2} \mathrm{O}_{3}$ example; and the anonymous referees for their helpful suggestions. 


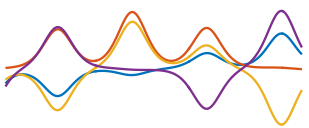

(a)

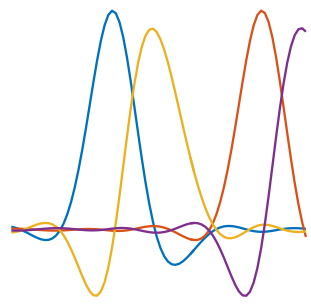

(c)

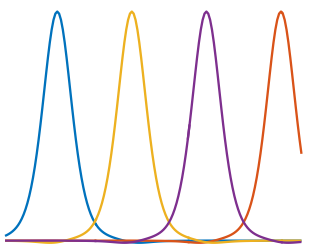

(b)

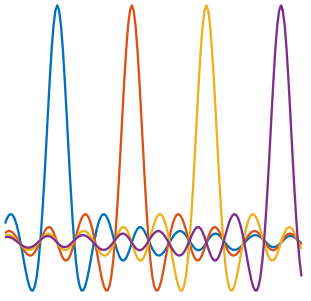

(d)

Fig. 9: For a simple one dimensional model problem, we plot the eigenfunctions (a) and Wannier functions for (b) the isolated case, (c) entangled case 1, and (d) entangled case 2 . Here, only a portion of the computational domain (horizontal axis) is shown to more clearly illustrate the local structure of the Wannier functions.

\section{REFERENCES}

[1] E. Anderson, Z. Bai, C. Bischof, S. Blackford, J. Demmel, J. Dongarra, J. Du Croz, A. Greenbaum, S. Hammarling, A. McKenney, and D. Sorensen, LAPACK Users' Guide, SIAM, Philadelphia, PA, third ed., 1999.

[2] M. Benzi, P. Boito, And N. Razouk, Decay properties of spectral projectors with applications to electronic structure, SIAM Rev., 55 (2013), pp. 3-64.

[3] E. I. Blount, Formalisms of band theory, Solid State Ohys., 13 (1962), pp. 305-373.

[4] D. R. Bowler AND T. MiYAzAKI, $O(N)$ methods in electronic structure calculations, Rep. Prog. Phys., 75 (2012), p. 036503.

[5] C. Brouder, G. Panati, M. Calandra, C. Mourougane, and N. Marzari, Exponential localization of Wannier functions in insulators, Phys. Rev. Lett., 98 (2007), p. 046402.

[6] P. Businger And G. H. Golub, Linear least squares solutions by householder transformations, Numerische Mathematik, 7 (1965), pp. 269-276.

[7] E. Cancès, A. Levitt, G. Panati, And G. Stoltz, Robust determination of maximallylocalized Wannier functions, Phys. Rev. B, 95 (2017), p. 075114.

[8] S. Chandrasekaran And I. C. IPSEn, On rank-revealing factorisations, SIAM J. on Matrix Anal. and Apps., 15 (1994), pp. 592-622.

[9] C.-Z. Chang, W. Zhao, D. Y. Kim, H. Zhang, B. A. Assaf, D. Heiman, S.-C. Zhang, C. LiU, M. H. Chan, AND J. S. Moodera, High-precision realization of robust quantum anomalous hall state in a hard ferromagnetic topological insulator, Nature materials, 14 (2015), p. 473.

[10] H. Cornean, D. Gontier, A. Levitt, And D. Monaco, Localised wannier functions in metallic systems, ArXiv e-prints, (2017), arXiv:1712.07954.

[11] H. D. Cornean, D. Monaco, and S. Teufel, Wannier functions and $\digamma_{2}$ invariants in time- 
reversal symmetric topological insulators, Rev. Math. Phys., 29 (2017), p. 1730001.

[12] A. Damle, L. Lin, AND L. Ying, Compressed representation of Kohn-Sham orbitals via selected columns of the density matrix, J. Chem. Theory Comput., 11 (2015), pp. 1463-1469.

[13] A. Damle, L. Lin, And L. Ying, Scdm-k: Localized orbitals for solids via selected columns of the density matrix, J. Comput. Phys., 334 (2017), pp. $1-15$.

[14] W. E, T. LI, AND J. LU, Localized bases of eigensubspaces and operator compression, Proc. Natl. Acad. Sci., 107 (2010), pp. 1273-1278.

[15] P. Giannozzi, S. Baroni, N. Bonini, M. Calandra, R. Car, C. Cavazzoni, D. Ceresoli, G. L. Chiarotti, M. Cococcioni, I. Dabo, A. D. Corso, S. de Gironcoli, S. Fabris, G. Fratesi, R. Gebauer, U. Gerstmann, C. Gougoussis, A. Kokalu, M. Lazzeri, L. Martin-Samos, N. Marzari, F. Mauri, R. Mazzarello, S. Paolini, A. Pasquarello, L. Paulatto, C. Sbraccia, S. Scandolo, G. Sclauzero, A. P. Seitsonen, A. Smogunov, P. Umari, And R. M. Wentzcovitch, QUANTUM ESPRESSO: a modular and open-source software project for quantum simulations of materials, J. Phys.: Condens. Matter, 21 (2009), pp. 395502-395520.

[16] S. Goedecker, Linear scaling electronic structure methods, Rev. Mod. Phys., 71 (1999), pp. $1085-1123$.

[17] G. H. Golub and C. F. Van Loan, Matrix computations, Johns Hopkins Univ. Press, Baltimore, fourth ed., 2013.

[18] M. Gu AND S. Eisenstat, Efficient algorithms for computing a strong rank-revealing qr factorization, SIAM J. Sci. Comput., 17 (1996), pp. 848-869.

[19] F. GyGI, Compact representations of Kohn-Sham invariant subspaces, Phys. Rev. Lett., 102 (2009), p. 166406.

[20] F. D. M. Haldane, Model for a quantum hall effect without landau levels: Condensed-matter realization of the "parity anomaly", Phys. Rev. Lett., 61 (1988), pp. 2015-2018.

[21] P. Hohenberg and W. Kohn, Inhomogeneous electron gas, Phys. Rev., 136 (1964), pp. B864B871.

[22] W. Humphrey, A. Dalke, and K. Schulten, VMD - Visual Molecular Dynamics, Journal of Molecular Graphics, 14 (1996), pp. 33-38.

[23] C. L. Kane and E. J. Mele, $Z_{2}$ topological order and the quantum spin hall effect, Phys. Rev. Lett., 95 (2005), p. 146802.

[24] E. KOCH AND S. GoedeCKer, Locality properties and Wannier functions for interacting systems, Solid State Commun., 119 (2001), p. 105.

[25] W. KoHn, Analytic properties of Bloch waves and Wannier functions, Phys. Rev., 115 (1959), p. 809.

[26] W. KoHn, Density functional and density matrix method scaling linearly with the number of atoms, Phys. Rev. Lett., 76 (1996), pp. 3168-3171.

[27] W. Kohn and L. Sham, Self-consistent equations including exchange and correlation effects, Phys. Rev., 140 (1965), pp. A1133-A1138.

[28] L. Lin, Localized spectrum slicing, Math. Comp., 86 (2017), p. 2345.

[29] P.-O. LöwDin, On the non-orthogonality problem connected with the use of atomic wave functions in the theory of molecules and crystals, J. Chem. Phys., 18 (1950), pp. 365-375.

[30] N. Marzari, A. A. Mostofi, J. R. Yates, I. Souza, and D. Vanderbilt, Maximally localized Wannier functions: Theory and applications, Rev. Mod. Phys., 84 (2012), pp. 1419-1475.

[31] N. MaRZARI AND D. VANDERBILt, Maximally localized generalized Wannier functions for composite energy bands, Phys. Rev. B, 56 (1997), p. 12847.

[32] H. J. Monkhorst and J. D. Pack, Special points for Brillouin-zone integrations, Phys. Rev. B, 13 (1976), p. 5188.

[33] A. Mostofi, J. Yates, Y. Lee, I. Souza, D. Vanderbilt, and N. Marzari, Wannier90: a tool for obtaining maximally-localised wannier functions, Comput. Phys. Commun., 178 (2008), p. 685.

[34] J. I. Mustafa, S. Coh, M. L. Cohen, and S. G. Louie, Automated construction of maximally localized Wannier functions: Optimized projection functions method, Phys. Rev. B, 92 (2015), p. 165134.

[35] G. Nenciu, Dynamics of band electrons in electric and magnetic fields: rigorous justification of the effective hamiltonians, Rev. Mod. Phys., 63 (1991), pp. 91-127.

[36] V. Ozolinsš, R. Lai, R. CAFlisch, And S. Osher, Compressed modes for variational problems in mathematics and physics, Proc. Natl. Acad. Sci., 110 (2013), pp. 18368-18373.

[37] G. Panati And A. Pisante, Bloch bundles, Marzari-Vanderbilt functional and maximally localized Wannier functions, Commun. Math. Phys., 322 (2013), pp. 835-875.

[38] J. P. Perdew, M. Ernzerhof, And K. Burke, Rationale for mixing exact exchange with density functional approximations, J. Chem. Phys., 105 (1996), pp. 9982-9985. 
[39] M. Reed And B. Simon, Methods of modern mathematical physics. IV. Analysis of operators, Academic Pr., 1978.

[40] A. A. Soluyanov and D. Vanderbilt, Wannier representation of $\digamma_{2}$ topological insulators, Phys. Rev. B, 83 (2011), p. 035108.

[41] I. Souza, N. Marzari, And D. VAnderbilt, Maximally localized wannier functions for entangled energy bands, Phys. Rev. B, 65 (2001), p. 035109.

[42] G. H. WANniER, The structure of electronic excitation levels in insulating crystals, Phys. Rev., 52 (1937), p. 191.

[43] J. R. Yates, X. Wang, D. Vanderbilt, and I. Souza, Spectral and Fermi surface properties from Wannier interpolation, Phys. Rev. B, 75 (2007), p. 195121. 